\title{
Machine learning model of the catalytic efficiency and specificity of acyl-ACP thioesterase variants generated by natural and in vitro directed evolution
}

\section{Fuyuan Jing}

lowa State University https://orcid.org/0000-0002-5281-6648

Keting Chen

lowa State University https://orcid.org/0000-0002-5242-7239

Marna Yandeau-Nelson

lowa State University https://orcid.org/0000-0002-2742-7384

Basil Nikolau ( $\square$ dimmas@iastate.edu )

lowa State University https://orcid.org/0000-0002-4672-7139

\section{Article}

Keywords: acid, acyl, fatty, important, directed, evolution, productivity

Posted Date: September 2nd, 2021

DOI: https://doi.org/10.21203/rs.3.rs-778286/v1

License: (c) (i) This work is licensed under a Creative Commons Attribution 4.0 International License.

Read Full License 


\section{Abstract}

Modulating the catalytic activity of acyl-ACP thioesterase (TE) is an important biotechnological target for effectively increasing flux and diversifying products of the fatty acid biosynthesis pathway. In this study, a directed evolution approach was developed to improve the fatty acid productivity and fatty acid diversity of E. coli strains expressing variant acyl-ACP TEs. A single round of directed in vitro evolution, coupled with a high-throughput colorimetric screen, identified 26 novel acyl-ACP TE variants, which convey up to 10-fold increase in fatty acid productivity, and altered fatty acid profiles when expressed in a bacterial host strain. These in vitro generated variant acyl-ACP TEs, in combination with 31 natural variants isolated from diverse phylogenetic origins were analyzed with a random forest classifier machine learning tool, generating a quantitative model that identified 22 amino acid residues, which define important structural features that determine the substrate specificity of acyl-ACP TE.

\section{Introduction}

Fatty acids, stored in the form of triacylglycerol in plant seeds, single cell microbes or animal adipocytes, are an abundant renewable resource as energy-dense, chemically highly-reduced forms of carbon. Societal consumption of these natural products occurs not only via the food supply, but also as industrial feedstocks of ingredients such as soaps, detergents, surfactants, lubricants, cosmetics, and pharmaceuticals ${ }^{1-3}$. With the rising cost of petroleum and growing environmental concerns about consuming large amounts of fossil carbon, the beginning of the 21 st century has seen increasing interest in using biological fatty acids for the production of biofuels or chemical feedstocks 4,5 .

In plants and bacteria, fatty acid biosynthesis is catalyzed by a Type II fatty acid synthase (FAS), using acetyl-CoA and malonyl-ACP as substrates. This process proceeds via the iterative cycle of four reactions (condensation-reduction-dehydration and reduction), which elongate the acyl-chain by 2-carbon atoms per cycle. The substrate intermediates throughout this process are esterified to the thiol group of a phosphopantetheinyl cofactor, carried by acyl carrier-protein (ACP). This elongation process can be terminated by either the transacylation of the acyl-chain to a glycerol backbone to begin the process of membrane glycerolipid assembly or by the hydrolysis of the thioester bond of acyl-ACP, catalyzed by acylACP thioesterase (TE), to release a free fatty acid. Many plant acyl-ACP TEs have been isolated and characterized, and they exhibit different fatty acyl chain length specificities, and thus play a crucial role in determining the chain lengths of the fatty acid products generated by plant and bacterial FAS systems ${ }^{6-}$ 9 .

Four oil seed crops (i.e., palm, soybean, canola, and sunflower) generate $80 \%$ of the world's 200 million metric tonnes of vegetable oils ${ }^{2}$. These oils serve as feedstocks for both dietary needs and as chemical precursors ${ }^{10}$. These fatty acids have relatively narrow chemical diversity, primarily providing fatty acids of 16- and 18-carbon chain lengths with different degrees of unsaturation (i.e., 0 to 3 carbon-carbon double bonds). In contrast, seeds of a few discrete phylogenetic plant clades (e.g., Cuphea, coconut and 
palm) are the source of the world's 12 million metric tonnes of lauric acid (a 12-carbon fatty acid) containing-oils, which are the feedstocks primarily for the soap and detergent industry ${ }^{11}$.

In more recent years, with the better understanding of the regulation of the fatty acid biosynthesis pathway, and the rapid advances in synthetic biology, there has been intense interest in the metabolic engineering of this pathway for the production of fatty acids of different chain lengths or different fatty acid derivatives $9,12-17$. These efforts have focused on increasing the chemical diversity of fatty acids and increasing fatty acid yields, and have concentrated on using three biological chassis: plant seeds ${ }^{18}$, bacteria ${ }^{9}$ and yeast ${ }^{19}$. Because of genetic tractability, the bacterial chassis has primarily focused on Escherichia coli, and two approaches for increasing productivity have been demonstrated, either independently or in combination. One is the overexpression of acyl-ACP TEs, which releases free fatty acids from the FAS system, and another is the elimination of the fatty acid $\beta$-oxidation pathway via mutations of either $\operatorname{fadD}$ (acyl-CoA synthetase) or fadE (acyl-CoA dehydrogenase) ${ }^{9}$.

The expression of acyl-ACP TEs in bacterial systems has two attributes. Based on the substrate specificity of the acyl-ACP TE that is used, one can control the acyl chain lengths of the fatty acids that the FAS system will produce 20,21 . In addition, expression of acyl-ACP TEs enhances fatty acid productivity of the strain by creating a new metabolic product-sink for the FAS pathway, and by depleting the in vivo long chain acyl-ACP pool size, which relieves feedback inhibition of upstream enzymes in the FAS pathway $9,22,23$. These attributes can be optimized by controlling the expression of acyl-ACP TES (e.g., using expression plasmid vectors with different promoter strengths, or different plasmid copy numbers) $17,24,25$. Thus, prior studies have used acyl-ACP TEs sourced from a variety of different natural sources, each of which have evolved for that organism's environmental niche. However, that natural evolutionary adaptation may not be optimal for the envisioned industrial application in the heterologous host, such as an E. coli strain bioengineered for fatty acid productivity or production of a fatty acid with a specific acyl chain length.

Limited structural information and a not well-understood catalytic mechanism for acyl-ACP TEs 26, 27 makes it challenging to increase the activity of this enzyme by rational design. Directed evolution, which mimics the natural evolutionary process at the lab scale, is a promising alternative approach, and provides a mechanism to identify and exploit genetic space that natural evolution may not have explored ${ }^{28-30}$. Directed evolution involves iterative rounds of random mutagenesis and screening for the desired biological properties. This strategy has been successfully applied on a number of biocatalysts to tailor their functions, including substrate specificity, catalytic turn-over, and thermostability $28,31,32$. In this study, directed evolution was undertaken to optimize the catalytic efficiency of acyl-ACP TE with the goal of improving fatty acid productivity in microbes.

Specifically, we selected six previously well-characterized plant acyl-ACP TEs as parental enzymes that display diverse catalytic efficiencies and substrate specificities ${ }^{21}$, and used a PCR-based approach to generate a library of acyl-ACP TE variants. In vivo screening this library of variants for individuals that 
express higher fatty acid productivity enabled the isolation and characterization of novel acyl-ACP TEs that exhibit improved catalytic efficiency as compared to the initial parental acyl-ACP TEs. These novel enzymes also express diverse substrate specificities relative to the acyl-chain length of the preferred acylACP substrate. Taking advantage of these acyl-ACP TE variants and other functionally characterized acylACP TEs reported in prior studies 21,33 , we implemented and optimized a random forest-directed machine learning approach to identify important residues that determine acyl-ACP TE substrate specificity.

\section{Methods}

\section{Design of mutagenesis oligonucleotides}

Amino acid sequences of the six acyl-ACP TEs that were used in this study are: CvFatB1 (AEM72522.1) and CvFatB2 (AEM72523.1) from Cuphea viscosissima; CnFatB2 (AEM72520.1) and CnFatB3 (AEM72521.1) from Cocos nucifera; UaFatB1 (AAB71731.1) from Ulmus Americana; and CpFatB1 (AAC49179.1) from Cuphea palustris. Supplemental Figure 1 shows comparisons of the sequences of these six mature TEs proteins (without the $\mathrm{N}$-terminal chloroplast targeting sequences). Random mutagenesis was used to generate 2-8 possible substitutions at 98 selected positions. These 98 positions were primarily selected for convenience in the design of the primers used to reassemble the acyl-ACP TE variant library. The variant library was generated by PCR reassembly of mutant acyl-ACP TEs by using 30 DNA oligonucleotide primers (labeled as M1-1 to M1-10, M2-1 to M2-10, and M3-1 to M3-10) that incorporated mixed nucleotides at each of the 98 selected positions (Supplemental Table 1). The ends of each of these 30 oligonucleotide primers overlapped with the adjoining oligonucleotide sequences by 22-25 nucleotides; the Tm values for these overlapping regions were in the range of 54$56^{\circ} \mathrm{C}$. In addition, the $5^{\prime}$ and $3^{\prime}$ ends of oligonucleotides M1-1 and M3-10 encoded BamHI and EcoRI restriction sites, respectively. These characteristics enabled subsequent PCR-based reassembly of the entire acyl-ACP TE sequence into a single DNA fragment, which contained terminal BamHI and EcoRI restriction sites for subsequent cloning purposes.

\section{PCR-Assembly of the variant acyl-ACP TE library}

The acyl-ACP TE-encoding variant library was generated by assembling the 30 oligonucleotide primers by two rounds of PCR. The first round of PCR was conducted in a $50 \mu \mathrm{L}$ reaction mix containing $0.15 \mu \mathrm{M}$ of each primer (primers M1-1 to M1-10, M2-1 to M2-10, and M3-1 to M3-10), commercial Taq PCR buffer (New England Biolabs, M0273), $0.4 \mathrm{mM}$ dNTP, $3 \mathrm{mM} \mathrm{MgCl}_{2}$, and 1 Unit of Taq DNA polymerase (New England Biolabs, USA). The thermal cycling program for the first round of PCR was initiated by incubating the mix at $95^{\circ} \mathrm{C}$ for $3 \mathrm{~min}$, and then 25 cycles of incubations at $95^{\circ} \mathrm{C}$ for $15 \mathrm{~s}, 50^{\circ} \mathrm{C}$ for $20 \mathrm{~s}$ and $68^{\circ} \mathrm{C}$ for $40 \mathrm{~s}$; the final extension step was at $68^{\circ} \mathrm{C}$ for $5 \mathrm{~min}$. Two- $\mu \mathrm{L}$ aliquots of product from the first round of PCR were used as the template for the second round of PCR. This second round of PCR consisted of a 50 $\mu \mathrm{L}$ reaction mixture containing $0.2 \mu \mathrm{M}$ of primer M1-1 and $0.2 \mu \mathrm{M}$ of primer M3-10), commercial Taq PCR buffer (New England Biolabs, M0273), $0.2 \mathrm{mM}$ dNTP, $1.5 \mathrm{mM} \mathrm{MgCl}_{2}$ and 1 Unit Taq DNA polymerase. The 
thermal cycling program began at $95^{\circ} \mathrm{C}$ for $3 \mathrm{~min}$, and then 28 cycles of $95^{\circ} \mathrm{C}$ for $15 \mathrm{~s}, 60^{\circ} \mathrm{C}$ for $20 \mathrm{~s}$ and $68^{\circ} \mathrm{C}$ for $40 \mathrm{~s}$, and a final 5-minute extension step at $68^{\circ} \mathrm{C}$.

Products from the second round of PCR were fractionated by electrophoresis in a 1\% agarose gel, and the 950 bp target band was purified with the QiaQuick gel extraction kit (Qiagen, Valencia, CA, USA). The recovered DNA was digested with $B a m H I$ and $E c o R I$, and cloned into the corresponding restriction sites of the vector, pUCHisGm (Supplemental Figure 2); this plasmid was specifically modified from pUC57 in this study. In this vector, the expression of the acyl-ACP TE sequence is under the transcriptional control of the lacZ promoter, and the acyl-ACP TE coding sequence is fused at the $\mathrm{N}$-terminus to a $6 \mathrm{x}$ His tag, and at the C-terminus to a gentamicin resistant gene $\left(\mathrm{Gm}^{\mathrm{R}}\right)$ that is separated from the acyl-ACP TE coding sequence via a dodecapeptide flexible linker-sequence, $\left[(\mathrm{Gly})_{3} \text {-Ser }\right]_{3}{ }^{34}$. The resulting mixture of plasmid vectors containing the variant acyl-ACP TE ORFs were transformed into E. coli K27 by electroporation. Hence, each recovered colony from this transformation event carried a plasmid that has the potential of expressing an individual variant acyl-ACP TE. As controls, the DNA fragments of the six mature wild-type acyl-ACP TE-coding sequences (UaFatB1, CpFatB1, CvFatB1, CvFatB2, CnFatB2, and CnFatB3) were also cloned into pUCHisGm and transformed into E coli strain K27.

\section{Colony screening of acyl-ACP TE variants by Neutral Red staining}

The initial screening of the acyl-ACP TE variants was conducted on solid media containing the $\mathrm{pH}$ indicator stain, Neutral Red. These 10-cm diameter Petri plates contained M9 minimal medium (50 mM $\mathrm{Na}_{2} \mathrm{HPO}_{4}, 20 \mathrm{mM} \mathrm{KH}_{2} \mathrm{PO}_{4}, 10 \mathrm{mM} \mathrm{NaCl}, 20 \mathrm{mM} \mathrm{NH}_{4} \mathrm{Cl}, 2 \mathrm{mM} \mathrm{MgSO}_{4}$, and $0.1 \mathrm{mM} \mathrm{CaCl}_{2}$ ) solidified with $15 \mathrm{~g} / \mathrm{L}$ agar and supplemented with $0.4 \%$ glucose, $100 \mathrm{mg} / \mathrm{L}$ carbenicillin, $2.5 \mathrm{mg} / \mathrm{L}$ gentamicin, $1 \mathrm{mM}$ isopropyl-B-D-thiogalactopyranoside (IPTG), and 100 ppm Neutral Red dye. Each Neutral Red plate was inoculated with an appropriate amount of the electroporation transformation mixture so that each plate supported the growth of $300-500$ colonies. Upon inoculation these plates were incubated at $30^{\circ} \mathrm{C}$ for three days, and colonies that showed the most intense red color were selected for further characterizations.

\section{Analysis of fatty acids by gas chromatography-mass spectrometry (GC-MS)}

Intensely red-staining colonies were selected from the Neutral Red plates, inoculated into $0.7 \mathrm{~mL}$ of LB medium supplemented with $100 \mathrm{mg} / \mathrm{L}$ carbenicillin, and cultured overnight at $30^{\circ} \mathrm{C}$ at a $250 \mathrm{rpm}$ agitation rate. $\mathrm{A} 0.1 \mathrm{~mL}$ aliquot of the overnight culture was used to inoculate $2 \mathrm{~mL} \mathrm{M} 9$ medium supplemented with $2 \%$ glucose, $100 \mathrm{mg} / \mathrm{L}$ carbenicillin and $0.1 \mathrm{mM}$ ITPG in $16-\mathrm{mL}$ culture-tubes. After incubating at $30^{\circ} \mathrm{C}$ with agitation at $250 \mathrm{rpm}$ for 48 hours, a $1.5 \mathrm{~mL}$ aliquot of the culture was used for fatty acid extraction. Namely, following the addition of $50 \mu$ g heptanoic acid (7:0), $50 \mu$ g undecanoic acid (11:0), and $100 \mu \mathrm{g}$ heptadecanoic acid (17:0) (Sigma-Aldrich, St. Louis, MO, USA) as internal standards, the mixture was acidified with $0.5 \mathrm{~mL}$ of $1 \mathrm{M} \mathrm{HCl}$, and $4 \mathrm{~mL}$ chloroform-methanol (1:1 vol/vol) was used to extract and recover the fatty acids from the culture. After vortexing for $10 \mathrm{~min}$, and centrifugation at $3000 \times \mathrm{g}$ for $4 \mathrm{~min}$, the lower chloroform phase was passed through an anhydrous $\mathrm{MgSO}_{4}$ column to remove trace amounts of water, and the volume of the recovered solution was reduced to $\sim 0.2 \mathrm{~mL}$ by 
evaporation under a stream of $\mathrm{N}_{2}$ gas. The samples were subjected to GC-MS analysis ${ }^{21}$. Controls were fatty acids extracted from $E$. coli cultures that harbored the non-modified pUCHisGm vector. The fatty acid titer produced by these control cultures was subtracted from the fatty acid titers produced by each acyl-ACP TE variant.

\section{Statistical analysis, random forest classification and model performance prediction}

Fatty acid yield and composition data obtained with each acyl-ACP TE variant were assessed by analysis of variance (ANOVA) and post-hoc Tukey's Honestly Significant Difference (HSD) tests using JMP, Version 15 (SAS Institute Inc., Cary, NC). Principal Component Analysis (PCA) was performed for fatty acid profiles using the prcomp () function in the R/stats package and $95 \%$ confidence ellipses were constructed using the dataEllipse() function in the R/car package ${ }^{35}$.

For the machine learning approach, the random forest classifier was applied to calculate the relative importance of individual amino acid residues in determining the substrate specificity of acyl-ACP TE ${ }^{36-38}$. The strategy used sequence variants of acyl-ACP TE enzymes whose in vivo catalytic capabilities were quantitatively evaluated in E. coli. Sequence variation between any two acyl-ACP TEs was represented as a vector using a binary scoring method, where the value " 0 " is assigned at an amino acid position if two acyl-ACP TEs have the same residue, and the value " 1 " is assigned if they have different residues at that position. The catalytic capability of each variant enzyme was scaled so that the average concentrations of all individual fatty acids was assigned the value of " 0 ", and the associated standard deviation was assigned the value of " 1 ". Ward's hierarchical clustering analysis ${ }^{39}$ was performed based on the Euclidean distances of scaled fatty acid profiles, using the hclust) function in the R/stat package (R core team, 2020). The resultant dendrogram was pruned to determine the enzyme cluster membership by cutreedynamic() function using method "hybrid" in R package "dynamicTreeCut" 40 . Any two TEs belonging to the same, hence derived cluster, were deemed to have similar substrate specificities and assigned the value " 0 ". Acyl-ACP TE pairs belonging to different clusters were categorized as having different substrate specificities and assigned the value " 1 ".

Random forest classification models were constructed using R package, "ranger" 41 , with the binary scores for the amino acid residues at each position as the predictors, and the binary scores of each enzyme's substrate specificity as the response variable. The training phase included the construction of 500 decision trees using gini impurity (i.e., the probability of misclassifying the substrate specificity relationship between two acyl-ACP TEs) as the node-split criteria for each tree ${ }^{42}$. The prediction of a random forest model is made by pooling the predictions from all trees. Feature importance scores for each residue position of the enzyme, including a randomly-generated position (i.e., a control feature), were calculated based on the total decrease in node gini-impurity averaged over the 500 trees. These calculations provide a quantitative measure of the importance of each residue in classifying the enzyme pairs into two classes, i.e., the pair of enzymes that each express the same or different catalytic capabilities. The importance scores and the associated p-values were calculated using the importance_pvalues() function in the "ranger" package. The p-values were corrected across all residues 
by controlling the false discovery rate $<5 \%{ }^{43}$. The random forest classification model was implemented ten times, and the average importance scores were calculated at each of the residue positions of the enzyme. The reported $p$-value for each position is presented as the maximum value of the ten models.

Subsequently, an incremental feature selection approach was used to identify the optimal random forest model comprised of the subset of residue positions that are critical in determining substrate specificity. Briefly, the residue positions were ranked in descending order based on the importance scores, and for incremental feature selection, an initial random forest model was constructed using the two residue positions with the highest importance scores as the predictors and the binary scores of substrate specificity as the responses. Additional models were subsequently constructed by iteratively adding one position based on the importance score rank to the initial model. We then applied a "leave-one-out" crossvalidation to evaluate the predictive performance of the models. The predictive performance of each model was evaluated by the metrics of recall, specificity, and Matthews Correlation Coefficient (MCC). We define the enzyme pairs displaying the same substrate specificity as a positive case, and the enzyme pairs displaying different substrate specificity as a negative case. The three evaluating metrics were calculated based on the number of true positives (TP), number of false negatives (FP), number of true negatives (TN), and number of false positives (FP) in a classification model, using the following formula:

$$
\begin{aligned}
& \text { Recall }=\frac{\mathrm{TP}}{\mathrm{TP}+\mathrm{FN}} \\
& \text { Specificity }=\frac{\mathrm{TN}}{\mathrm{TN}+\mathrm{FP}} \\
& \mathrm{MCC}=\frac{(\mathrm{TP} \times \mathrm{TN})-(\mathrm{FP} \times \mathrm{FN})}{\sqrt{(\mathrm{TP}+\mathrm{FP})(\mathrm{TP}+\mathrm{FN})(\mathrm{TN}+\mathrm{FP})(\mathrm{TN}+\mathrm{FN})}}
\end{aligned}
$$

This incremental feature selection calculation was iterated 20 times, and the average values of recall, specificity, and MCC were deduced. The model with the highest MCC value is considered as the optimal model, and the residue positions included in this model were identified as the most significant residues that influence the enzyme's substrate specificity.

\section{Results}

\section{Sequence polymorphisms encompassed in the acyl-ACP TE variant library}

Six parental acyl-ACP TEs (i.e., CnFatB3, CvFatB1, CnFatB2, UaFatB1, CvFatB2, and CpFatB1) (Supplemental Figure 1) were selected to initiate this directed evolution study because prior characterizations had identified that these enzymes express diverse substrate specificities and diverse in vivo fatty acid productivity when expressed in E. coli ${ }^{21}$. The directed evolution strategy that we implemented generated variant enzymes that were initially screened for enhanced fatty acid productivity in E. coli. Ninety-eight sequence polymorphisms that occur among the six parental acyl-ACP TEs were 
randomly recombined in vitro by a PCR-based reassembly of the acyl-ACP TE-coding sequences (See Methods).

An initial pilot study evaluated the diversity of the acyl-ACP TE sequences recoverable from the constructed variant library. As a control experiment, 47 colonies were randomly chosen from the initial transformants without the Neutral Red selection for enhanced fatty acid accumulation, and the acyl-ACP TE sequences were determined from the recovered plasmids. The sequences of these 47 variant acyl-ACP TEs all differ from each other and from the six parental acyl-ACP TE sequences that went into the design of the variant library. However, only two of the reassembled acyl-ACP TE sequences encode a fully translatable full length acyl-ACP TE protein. The majority of the recovered mutants in this small subsample contained nonsense mutations (e.g., premature stop codon), or frame shifts due to an insertion or deletion of a single nucleotide. These are likely due to mis-alignments during PCR assembly or errors introduced during the chemical synthesis of the oligomers that were used in the construction of the variant library.

\section{Neutral Red colony-staining screen to identify hyperactive acyl-ACP TEs}

Prior studies established that the host used to propagate the variant acyl-ACP TE library, E. coli strain K27, which carries a mutation in acyl-CoA synthetase $(f a d D)$, results in the over-production of free fatty acids ${ }^{20}$. Indeed, when expressed in this strain there is a direct relationship between the levels of acyl-ACP TE activity and the accumulation of free fatty acids ${ }^{33,44}$. Therefore, the acyl-ACP TE variant library was bulk screened by growing transformants on media plates supplemented with the $\mathrm{pH}$ indicator dye, Neutral Red. Because the higher accumulation of free fatty acids acidifies the media, the Neutral Red dye is a gauge of fatty acid productivity by individual colonies. Figure 1a shows colonies on a typical Neutral Redcontaining plate. The majority of the recovered colonies ( 98\%) displayed a light red/pink color, but about $2 \%$ of the colonies exhibited a more intensive red color, indicative of increased fatty acid production resulting in acidification.

Based on this rationale, we selected 133 dark red-staining colonies and 77 light red/pink colored colonies and determined the fatty acid productivity of these strains. Among the 133 dark red staining strains, $75 \%$ produced more than $600 \mu \mathrm{M}$ of fatty acids, $50 \%$ produced more than $1000 \mu \mathrm{M}$ of fatty acids, and $25 \%$ produced even more, reaching levels greater than $1200 \mu \mathrm{M}$ of fatty acids (Figure 1b). In contrast, the majority of the strains identified as light red/pink colored colonies produced $<100 \mu \mathrm{M}$ of fatty acids; the maximum amount of fatty acid produced by these light red/pink colonies was $260 \mu \mathrm{M}$ (Figure 1b). These results confirm that there is correlation between the intensity of the color produced by Neutral Red staining of colonies and the fatty acid productivity of these strains.

Ultimately, approximately 30,000 colonies were screened, which resulted in the selection of 480 strains that were expected to express a higher fatty acid productivity based on enhanced Neutral Red staining (Supplemental Table 2). The fatty acid productivity of these strains was determined and compared to the productivity of strains expressing the original six parental acyl-ACP TEs that were used 
as guides for the design of the acyl-ACP TE variant library. The fatty acid productivity of the strains expressing these parental acyl-ACP TEs range between $100 \mu \mathrm{M}$ to $900 \mu \mathrm{M}$ (Figure 2, green data bars). Among the 480 colonies that were selected with the Neutral Red colony-staining assay, 151 expressed a fatty acid productivity that is higher than $600 \mu \mathrm{M}$, ranging up to a maximum of $1700 \mu \mathrm{M}$ (Supplemental Table 3). These productivities are between 4- and 15-fold higher than five of the six parental acyl-ACP TEs. Even compared to the most productive parental acyl-ACP TE (i.e., CpFatB1), the productivities expressed by the variant acyl-ACP TEs are nearly 2-fold higher (Figure 2).

\section{Sequences of acyl-ACP TE variants}

The 175 acyl-ACP TE variants that expressed higher in vivo fatty acid productivities (ranging between 500 $\mu \mathrm{M}$ and $1700 \mu \mathrm{M}$ ) were sequenced. These sequences identified 26 distinct acyl-ACP TE variant proteins (Supplemental Figure 3). One of these variant proteins, TEGm162, recurred 147 times in the sequenced collection, TEGm204 was recovered 3 times, and TEGm198 was recovered twice; the remaining 22 sequences occurred uniquely in this collection (Supplemental Tables 2 and 3). None of these recovered sequences identified with the Neutral Red staining screen were included among the original 47 randomly selected control sequence variants, which were isolated without the Neutral Red-staining screen. Hence, these findings indicate that the Neutral Red staining screen has strong selection capability for acyl-ACP TE variants that express higher productivities of fatty acids. The collective average of the fatty acid productivity of the 147 independently isolated TEGm162 variants was $1170 \pm 210 \mu \mathrm{M}$, and the average for the three TEGm204 variants was $1100 \pm 140 \mu \mathrm{M}$. These productivities are $\sim 30 \%$ higher than the most productive parental acyl-ACP TE (i.e. CpFatB1), and 10-fold higher than the productivity of the least effective parental acyl-ACP TE (i.e., CnFatB3).

The 26 distinct acyl-ACP TE variant sequences selected by this directed evolution strategy (Supplemental Figure 3) were compared to each other and to the six parental acyl-ACP TEs that were used to initiate this study. These analyses demonstrate that the recovered acyl-ACP TE variants share an overall sequence identity of $\sim 67 \%$. Among the 307 amino acid positions of these recovered variant enzymes, polymorphisms occur at 100 positions, which is very close to the number of positions (98) we targeted for mutagenesis in the design of the variant library. The two additional polymorphic positions may be attributable to variants introduced by errors in DNA primer synthesis or by PCR errors.

Hierarchical clustering analysis of these variant sequences identify a majority clade that is most similar to two of the parental sequences, CvFatB1 and CpFatB1 (Figure 3a). Within this clade, variants TEGm413 and TEGm419 are closest in sequence to the CpFatB1 and CvFatB1 parents, and these four proteins share $\sim 64 \%$ amino acid identity, but they express in vivo fatty acid productivities that range between $\sim 240 \mu \mathrm{M}$ and $\sim 1390 \mu \mathrm{M}$ (Figure 3b).

\section{The substrate specificities of acyl-ACP TE variants}

In addition to showing differences in in vivo fatty acid productivity, the six parental acyl-ACP TEs that were used to guide this directed evolution strategy also display differences in acyl-chain length substrate 
specificity. This variation provided an added opportunity to explore the relationship between amino acid sequence and substrate specificity attributes of acyl-ACP TEs. Therefore, we evaluated how substrate specificity evolved in the acyl-ACP TE variants that were selected for enhanced in vivo fatty acid productivity.

Figure $3 \mathrm{~b}$ shows the fatty acid profiles produced by the 26 evolved acyl-ACP TE variants as compared to the six parental acyl-ACP TEs. Prior characterizations of the six parental acyl-ACP TEs, in the context of the structural difference among 31 naturally occurring diverse acyl-ACP TEs from plant and microbial sources, had categorized these parental enzymes into three classes, Class I to III ${ }^{21}$. CvFatB2 and CnFatB2 are Class I enzymes that primarily hydrolyze acyl-ACPs of 14- and 16-carbon fatty acyl chains, CnFatB3 is a Class II enzyme that prefers 8- to 16-carbon acyl-chains, and CpFatB1, CvFatB1, and UaFatB1 are Class III enzymes that have a preference for 8-carbon acyl-chains ${ }^{21}$. The 26 acyl-ACP TE variants generated by this directed evolution study distributed somewhat unevenly among these three functional classes, with a preference for Class I and Class II enzymes (13 and 9 variants, respectively), and only four variants (TEGm162, TEGm169, TEGm258, and TEGm288) belonging to Class III acyl-ACP TEs. Although these 26 variant acyl-ACP TEs are classifiable among these categories, an analysis of variance (ANOVA) demonstrates that these substrate specificity classifications do not explain the observed variations in the in vivo fatty acid productivity observed in the $E$. coli host ( $p$-value $>0.05$ ).

\section{Machine learning model reveals structural constraints to substrate specificity.}

Because acyl-ACP TE classification based solely on sequence similarity and diversity does not fully predict the fatty acid productivity of these enzymes, we adopted an alternative classification strategy based on the fatty acid profiles. Thus, in addition to clustering the variant acyl-ACP TEs relative to their sequence similarity (Figure 3a), clustering was performed based on the fatty acid profiles produced when variant enzymes were expressed in vivo to evaluate their substrate specificity. These analyses not only evaluated the acyl-ACP TEs generated by the current in vitro directed evolution study, but also included previously characterized natural variants of acyl-ACP TE isolated from a wide variety of different phylogenetic clades ${ }^{21,33}$. Thus, collectively 57 acyl-ACP TE variants were analyzed, 26 being products of in vitro directed evolution selection and 31 being products of natural evolution selection. Hierarchical clustering that minimized within-cluster variance in substrate specificity separated the 57 acyl-ACP TE variants into three distinct clusters (Clusters A-C) (Figure 4a). Similar segregation pattern occurs upon principal component analysis (PCA) of these data (Figure $4 \mathrm{~b}$ ), and in combination the two primary principal components (PC1 and PC2) explain nearly $60 \%$ of the variation in the substrate specificity among these variants. $\mathrm{PC} 1$, which accounts for $43 \%$ of the variation in the fatty acids profiles, primarily separates Cluster C-enzymes from Clusters A and B, while PC2 explains $16 \%$ of the variation, and separates Cluster A from Cluster B and Cluster C (Figure $4 b$ ). This tripartite classification of the variants reflects the prior classification of naturally occurring acyl-ACP TEs variants ${ }^{21}$, which identified three classes of acyl-ACP TEs, with preferences for C14/C16 (Class I), C8 (Class III) or broad range chain-length (Class II) acyl-ACP TE substrates. Similarly in this study, Cluster A and Cluster C enzymes exhibit 
preferences for C8 and C14/C16 acyl-ACPs, respectively, whereas Cluster B enzymes have broader substrate specificity able to hydrolyze C8 to C16 acyl-ACPs (Figure 4c, 4d, 4e).

Manual comparisons of the recovered acyl-ACP TE sequence variants and their substrate specificities can provide constraints on the relationship between primary structure and substrate specificity of these enzymes. For example, by comparing the acyl-ACP TE sequence variants that are sorted into the same sequence-based hierarchical cluster, but are separated into different functional classes based on substrate specificities (i.e., Classes A-C; Figure 4a), one can heuristically identify those polymorphic residues that contribute to altered substrate specificity. We however, developed a systematic computational machine learning random forest classification model that improves on this manual strategy, and quantitatively assessed the importance of each polymorphic amino acid residue in determining the substrate specificity of the acyl-ACP TE variants.

The random forest classification strategy utilized both binarized substrate specificity data and amino acid sequence data as described in the Methods. Substrate specificity was binarized according to the fatty acids produced when each variant enzyme was expressed in E. coli, and two acyl-ACP TEs were defined as sharing substrate specificity if they were members of the same Cluster (A, B or C) (Figure 4a). In juxtaposition, two acyl-ACP TEs that had membership in separate Clusters were deemed as having different substrate specificities. After transforming the data and encoding, a random forest classifier was trained with all encoded data, and the mean feature importance scores for the 350 amino acid positions were calculated based on ten iterations of the model (Figure 5a and Supplemental Table 4a), which quantified the importance of individual residues in determining substrate specificity of each acyl-ACP TE variants. A total of 174 residue positions with importance scores ranging from $\sim 0.5$ to $\sim 15$, had a statistically significant impact on substrate specificity (i.e. corrected p-values $<0.001$; Supplemental Table 4a), and these are blue-highlighted in Figure 5a.

This list of residues was refined by a two-step approach. Initially, an incremental feature selection (IFS) approach was used that built a series of random forest models, in which each model added an additional residue to the evaluation process. The random forest classifier that included the 59 residue positions with the highest importance scores as the predictors exhibited optimal predictive performance, with a recall (i.e., true positive rate) of $70 \%$, a specificity (true negative rate) of $91 \%$, and a MCC of 0.69 (Figure $5 \mathrm{~b}$; Supplemental Table $4 \mathrm{a}$ ). Next, the list of 59 residues were further refined by pairwise comparisons of MCC scores using Student's t-test between every pair of adjacent models (i.e., the model that included one additional residue position versus the previous model that did not include that additional residue) until all 59 residues were examined (Figure $5 b$ and $5 c$ ). The final model that contained the top 22 residue positions (orange-highlighted in Figure $5 \mathrm{a}$ ) reached the statistical plateau of MCC (q-value $>0.05$; Supplemental Table $4 \mathrm{~b}$ ), and thus these 22 residues were considered as most impactful in determining the substrate specificity of the enzyme.

Mapping these 22 residues onto a predicted three dimensional structure of CvFatB2 indicates that the majority of these residues (17 of 22) are located in the N-terminal hot-dog domain structure (Figure 6). 
The other five residues are in the C-terminal hot-dog domain structure, among which are two residues that are adjacent to the catalytic residues we identified in a previous study 27 .

\section{Discussion}

Human civilization has been enabled by our ability to harness and uniquely utilize outputs from biological systems ${ }^{45}$. Via the domestication of animal, plant and microbial life forms ${ }^{46}$, we have developed technologies that support the ability of the human species to colonize nearly all niches that are available on the earth, and we are now contemplating technologies to colonize niches beyond the confines of our planet. Paramount to the growth of human civilization has been the ability to have ample food-supply and the ability to harness energy from the environment that supports these activities. Since the start of the industrial revolution in the 18th century, with the invention of the steam engine, we have become increasingly dependent on the oxidation of fossil carbon, first in the form of coal, and subsequently liquid (i.e., oil) and gaseous (i.e., natural gas) forms of fossil carbon. In parallel to these energy-generating carbon-oxidation processes, we have developed technologies that convert fossil carbon to materials that support our modern forms of life (i.e., the petrochemical industry).

Over the past 250-years, these activities have increasingly disrupted the earth's ecological carbon-balance that has taken millions of years to reach equilibrium. Thus, we now face the increasing challenge of carbon in the atmosphere $\left(\mathrm{CO}_{2}, \mathrm{CO}, \mathrm{CH}_{4}\right)$, which contributes to global warming and climate change, and the earth's land and ocean environments are increasingly polluted by non-degradable carbon polymers (e.g., single use plastics). In response there has been increasing research to adapt or engineer biological systems as platforms for generating biorenewable chemicals or biofuels generated from photosynthetically fixed $\mathrm{CO}_{2}$. Although global biological photosynthesis can fix sufficient quantities of atmospheric $\mathrm{CO}_{2}$ to meet current needs for fuels and chemicals, most of that biological carbon occurs in the form of lignocellulosic material. But unlike fossil carbon, which is chemically highly reduced, lacking oxygen, and is thus energy dense, lignocellulosic carbon is partially oxidized, and thus has lower energy density.

Fatty acids in contrast, contain less oxygen and are energy dense, and are therefore more similar to fossil carbon feedstocks, particularly petroleum. Therefore, there's been considerable interest in converting lignocellulosic carbon (e.g., sugars) to fatty acids, chemically removing oxygen and increasing the energy density of the product. A number of microbial chassis have been proposed for this conversion, including bacteria and yeast 9,47 . One of the key biocatalysts that has been the focus of these conversion processes is, acyl-ACP TE, the enzyme that intercepts and terminates the process of fatty acid biosynthesis by hydrolyzing the fatty acid product from the FAS enzyme, and thus generating a new product sink for this process. This bioengineering strategy releases the FAS system from the "normal" regulatory circuit that controls the fatty acid productivity of the chassis, resulting in the over-production of fatty acids. 
In this study, we developed a facile, directed evolution strategy to generate novel acyl-ACP TEs for the purpose of enhancing the fatty acid productivity in E. coli. The effectiveness of this strategy is exemplified by the fact that just a single round of directed evolution, and screening only a limited number of variants (i.e., 30,000), resulted in the isolation of 26 distinct acyl-ACP TE that enhanced fatty acid productivity in the model chassis by up to 10 -fold, as compared to the initial parental enzymes that constrained the in vitro directed evolution strategy. Using bacterial strains that harbor such an enhanced biocatalyst improved the efficiency of the conversion of glucose to fatty acids.

While the improvement of enzymatic activity is an important target for using acyl-ACP TE enzymes to hyper-produce fatty acids, this enzyme's substrate specificity is another significant attribute that can be bioengineered because it determines the chain length of fatty acids produced by a microbial chassis. This latter trait is important in determining the "performance" of the fatty acid products in the application arena, which is a prominent determinant for the market niche of these fatty acid products.

Although the directed evolution strategy was designed to improve fatty acid productivity of the microbial chassis by evolving acyl-ACP TE, the recovered enzymes also diversified the fatty acids produced by the microbial chassis. These data therefore provided an opportunity to explore the relationship between protein sequence and substrate specificity. Therefore, we integrated data from the 26 variant enzymes generated within this study, with data previously generated from 31 naturally occurring variant enzymes isolated from plants and bacteria ${ }^{21}$. Using fatty acid profile data combined from these 57 variant enzymes, a random forest classification algorithm systematically assessed the impact of each acyl-ACP TE residue on determining the substrate specificity of the enzyme. Such random forest strategies have proven useful in quantitatively modeling relationships between protein sequence and different protein functionalities, including protein folding and crystallizability ${ }^{48-50}$.

Collectively, these analyses assigned an importance score to each residue in its ability to affect a change in substrate specificity of the enzyme. Twenty two of the most significant contributors in determining the substrate specificity of the enzyme were identified. Six of these residues had previously been identified in the CvFatB2 enzyme (i.e., V115, N121, R124, R125, L155, and 1166 of the CvFatB2 sequence) via a domain shuffling strategy and confirmed by site-directed mutagenesis studies as being critical in determining substrate specificity ${ }^{51}$.

The majority of highest scoring residues (17 out of 22 residues) reside within the N-terminal hot-dog structure of the predicted tertiary structure model of CvFatB2a (Fig. 6). This location is consistent with our prior postulate ${ }^{51}$ that the substrate specificity of this enzyme is determined by the chemophysical nature of the substrate binding pocket located in the N-terminal hot-dog domain of these enzymes; the substrate binding pocket being formed between the central a-helix and the antiparallel $\beta$-sheets in the $\mathrm{N}$ terminal hotdog domain ${ }^{51}$. Specifically, the active site residues of acyl-ACP TE are located on the Cterminal hot-dog structure at the interface between the N-terminal and C-terminal hot-dog structures. While four of these residues (i.e., V115, N121, R124, and R15) that are located on the antiparallel $\beta$-sheets had previously been identified as being important in determining substrate specificity, five additional 
amino acids (i.e., I52, L59, A63, L64, and V67) located on the central a-helix of the N-terminal hot-dog structure have been identified by the current machine learning strategy. Moreover, a few pairs of evolved acyl-ACP TE variants that show different substrate specificities display only a small number of polymorphic amino acid residues. This further indicates the importance of these residues in determining the substrate specificity of acyl-ACP TE. By examining the positions of the 22 most significant residues identified by machine learning (Fig. 6), we hypothesize that the residues on the central a-helix and the antiparallel $\beta$-sheets determine the substrate specificity of acyl-ACP TE by defining the size and chemophysical property of the substrate binding pocket. Other residues identified (i.e., V26, D29, N74, Y84, D87, N100, and A192) are located at the opening of the active site cleft near the surface of the acylACP TE protein and they may affect the substrate specificity by modulating the interactions between the enzyme and the ACP moiety of the substrate. Indeed, we had previously identified residues at the surface of the acyl-ACP TE enzyme that are important for protein-protein interaction and thus affect catalytic efficiency and substrate specificity of acyl-ACP TE ${ }^{51}$.

Collectively, the findings presented herein provide an experimentally-based computational model that pinpoints amino acid residues that determine the substrate specificity of acyl-ACP TEs. This study demonstrates the feasibility of combining an in vitro directed evolution approach with downstream computational analysis to identify key structural features (i.e., amino acid residues) of an enzyme that can be targeted in a rational redesign strategy to further enhance the productivity and specificity of a microbial biofactory. Hence, one can envision that a rational design strategy, guided by results of a directed evolution study, which does not require any a priori knowledge of sequence-activity relationships, would be even more powerful in affecting desirable improvements in biocatalysis, and thereby enabling advances in biomanufacturing of a target biochemical. In this study, we demonstrate an integrated directed evolution-machine learning strategy that has been used to understand the structural features of the protein that contribute to increasing the catalytic efficiency of acyl-ACP TE and further expand the knowledge on the structural determinants of the substrate specificity of this enzyme. Such a strategy enables the alteration of two attributes of this important biocatalyst and its utilization to build an efficient biosynthetic pathway for producing desired fatty acids as feedstocks for biorenewable chemicals.

\section{Declarations}

\section{Acknowledgements}

This work was partially funded by the U.S. National Science Foundation through its Engineering Research Center Program (Award No. EEC-0813570) in support of the Engineering Research Center for Biorenewable Chemicals; CBiRC. The authors thank Dr. M. Ann D. N. Perera and Dr. Zhihong Song of lowa State University's W. M. Keck Metabolomics Research Laboratory for assistance with fatty acid analysis. We thank Sara Pederson for helping with the mutant library generation and fatty acid analysis. We thank Dr. Le Zhao for picking colonies and making glycerol frozen stocks. We thank Derek Loneman for assistance with fatty acid extraction. 


\section{References}

1. Thelen, J.J. \& Ohlrogge, J.B. Metabolic engineering of fatty acid biosynthesis in plants. Metabolic Engineering 4, 12-21 (2002).

2. Dyer, J.M., Stymne, S., Green, A.G. \& Carlsson, A.S. High-value oils from plants. Plant Journal 54, 640-655 (2008).

3. Ohlrogge, J.B. Design of New Plant Products: Engineering of Fatty Acid Metabolism. Plant Physiology 104, 821-826 (1994).

4. Durrett, T.P., Benning, C. \& Ohlrogge, J. Plant triacylglycerols as feedstocks for the production of biofuels. Plant Journal 54, 593-607 (2008).

5. Nikolau, B.J., Perera, M.A., Brachova, L. \& Shanks, B. Platform biochemicals for a biorenewable chemical industry. Plant J 54, 536-545 (2008).

6. Voelker, T.A. et al. Fatty acid biosynthesis redirected to medium chains in transgenic oilseed plants. Science 257, 72-74 (1992).

7. Leonard, J.M., Knapp, S.J. \& Slabaugh, M.B. A Cuphea beta-ketoacyl-ACP synthase shifts the synthesis of fatty acids towards shorter chains in Arabidopsis seeds expressing Cuphea FatB thioesterases. Plant J 13, 621-628 (1998).

8. Pollard, M.R., Anderson, L., Fan, C., Hawkins, D.J. \& Davies, H.M. A specific acyl-ACP thioesterase implicated in medium-chain fatty acid production in immature cotyledons of Umbellularia californica. Archives of Biochemistry and Biophysics 284, 306-312 (1991).

9. Lennen, R.M. \& Pfleger, B.F. Engineering Escherichia coli to synthesize free fatty acids. Trends Biotechno/ 30, 659-667 (2012).

10. Patel, M. et al. (Utrecht University, Department of Science, Technology and Society (STS ...

11. Knaut, J. \& Richtler, H. Trends in industrial uses of palm and lauric oils. Journal of the American Oil Chemists' Society 62, 317-327 (1985).

12. Magnuson, K., Jackowski, S., Rock, C.O. \& Cronan, J.E., Jr. Regulation of fatty acid biosynthesis in Escherichia coli. Microbiological reviews 57, 522-542 (1993).

13. Handke, P., Lynch, S.A. \& Gill, R.T. Application and engineering of fatty acid biosynthesis in Escherichia coli for advanced fuels and chemicals. Metabolic Engineering 13, 28-37 (2011).

14. Liu, X., Sheng, J. \& Curtiss, R., 3rd Fatty acid production in genetically modified cyanobacteria. Proceedings of the National Academy of Sciences of the United States of America 108, 6899-6904 (2011).

15. Zhang, X.J., Li, M., Agrawal, A. \& San, K.Y. Efficient free fatty acid production in Escherichia coli using plant acyl-ACP thioesterases. Metabolic Engineering 13, 713-722 (2011).

16. Ranganathan, S. et al. An integrated computational and experimental study for overproducing fatty acids in Escherichia coli. Metabolic Engineering 14, 687-704 (2012). 
17. Zhang, F., Carothers, J.M. \& Keasling, J.D. Design of a dynamic sensor-regulator system for production of chemicals and fuels derived from fatty acids. Nature Biotechnology 30, 354-359 (2012).

18. Dehesh, K., Jones, A., Knutzon, D.S. \& Voelker, T.A. Production of high levels of 8:0 and 10:0 fatty acids in transgenic canola by overexpression of Ch FatB2, a thioesterase cDNA from Cuphea hookeriana. Plant J 9, 167-172 (1996).

19. Gajewski, J., Pavlovic, R., Fischer, M., Boles, E. \& Grininger, M. Engineering fungal de novo fatty acid synthesis for short chain fatty acid production. Nat Commun 8, 14650 (2017).

20. Voelker, T.A. \& Davies, H.M. Alteration of the specificity and regulation of fatty acid synthesis of Escherichia coli by expression of a plant medium-chain acyl-acyl carrier protein thioesterase. $J$ Bacteriol 176, 7320-7327 (1994).

21. Jing, F. et al. Phylogenetic and experimental characterization of an acyl-ACP thioesterase family reveals significant diversity in enzymatic specificity and activity. BMC biochemistry 12, 44 (2011).

22. Heath, R.J. \& Rock, C.O. Inhibition of beta-ketoacyl-acyl carrier protein synthase III (FabH) by acyl-acyl carrier protein in Escherichia coli. J Biol Chem 271, 10996-11000 (1996).

23. Jiang, P. \& Cronan, J.E. Inhibition of Fatty-Acid Synthesis in Escherichia-Coli in the Absence of Phospholipid-Synthesis and Release of Inhibition by Thioesterase Action. Journal of Bacteriology 176, 2814-2821 (1994).

24. Steen, E.J. et al. Microbial production of fatty-acid-derived fuels and chemicals from plant biomass. Nature 463, 559-562 (2010).

25. Youngquist, J.T. et al. Kinetic modeling of free fatty acid production in Escherichia coli based on continuous cultivation of a plasmid free strain. Biotechnol Bioeng 109, 1518-1527 (2012).

26. Feng, Y. et al. Structural insight into acyl-ACP thioesterase toward substrate specificity design. ACS chemical biology 12, 2830-2836 (2017).

27. Jing, F., Yandeau-Nelson, M.D. \& Nikolau, B.J. Identification of Active Site Residues Implies a Twostep Catalytic Mechanism for Acyl-ACP Thioesterase. Biochemical Journal, BCJ20180470 (2018).

28. Turner, N.J. Directed evolution drives the next generation of biocatalysts. Nat Chem Bio/ 5, 568-574 (2009).

29. Cobb, R.E., Sun, N. \& Zhao, H.M. Directed evolution as a powerful synthetic biology tool. Methods 60, 81-90 (2013).

30. Dougherty, M.J. \& Arnold, F.H. Directed evolution: new parts and optimized function. Curr Opin Biotech 20, 486-491 (2009).

31. Zha, W.J., Rubin-Pitel, S.B. \& Zhao, H.M. Exploiting genetic diversity by directed evolution: molecular breeding of type III polyketide synthases improves productivity. Mol Biosyst 4, 246-248 (2008).

32. Nair, N.U. \& Zhao, H.M. Evolution in reverse: Engineering a D-xylose-specific xylose reductase. Chembiochem 9, 1213-1215 (2008). 
33. Jing, F., Zhao, L., Yandeau-Nelson, M.D. \& Nikolau, B.J. Two distinct domains contribute to the substrate acyl chain length selectivity of plant acyl-ACP thioesterase. Nature communications 9,860 (2018).

34. Chen, X., Zaro, J.L. \& Shen, W.C. Fusion protein linkers: Property, design and functionality. Adv Drug Deliv Rev (2012).

35. Fox, J. \& Weisberg, S. An R Companion to Applied Regression. (SAGE Publications, 2011).

36. Basu, S., Soderquist, F. \& Wallner, B. Proteus: a random forest classifier to predict disorder-to-order transitioning binding regions in intrinsically disordered proteins. J Comput Aided Mol Des 31, 453466 (2017).

37. Wang, M. et al. FunSAV: predicting the functional effect of single amino acid variants using a twostage random forest model. PloS one 7, e43847 (2012).

38. Luttrell, J., Liu, T., Zhang, C. \& Wang, Z. Predicting protein residue-residue contacts using random forests and deep networks. BMC bioinformatics 20, 100 (2019).

39. Ward, J.H. Hierarchical Grouping to Optimize an Objective Function. Journal of the American Statistical Association 58, 236-244 (1963).

40. Langfelder, P. \& Horvath, S. WGCNA: an R package for weighted correlation network analysis. $B M C$ Bioinformatics 9, 559 (2008).

41. Wright, M.N. \& Ziegler, A. ranger: A Fast Implementation of Random Forests for High Dimensional Data in C + + and R. 201777, 17 (2017).

42. Guyon, I. \& Elisseeff, A. An introduction to variable and feature selection. Journal of machine learning research 3, 1157-1182 (2003).

43. Benjamini, Y. \& Hochberg, Y. Controlling the False Discovery Rate: A Practical and Powerful Approach to Multiple Testing. Journal of the Royal Statistical Society: Series B (Methodological) 57, 289-300 (1995).

44. Jing, F., Yandeau-Nelson, M.D. \& Nikolau, B.J. Identification of active site residues implies a two-step catalytic mechanism for acyl-ACP thioesterase. Biochem J 475, 3861-3873 (2018).

45. Holdren, J.P. \& Ehrlich, P.R. Human Population and the Global Environment: Population growth, rising per capita material consumption, and disruptive technologies have made civilization a global ecological force. American Scientist 62, 282-292 (1974).

46. Stetter, M.G., Gates, D.J., Mei, W. \& Ross-Ibarra, J. How to make a domesticate. Curr Biol 27, R896R900 (2017).

47. Leber, C. \& Da Silva, N.A. Engineering of Saccharomyces cerevisiae for the synthesis of short chain fatty acids. Biotechnol Bioeng 111, 347-358 (2014).

48. Jo, T. \& Cheng, J. in BMC bioinformatics, Vol. 15 S14 (Springer, 2014).

49. Jahandideh, S. \& Mahdavi, A. RFCRYS: Sequence-based protein crystallization propensity prediction by means of random forest. Journal of theoretical biology 306, 115-119 (2012). 
50. Bonetta, R. \& Valentino, G. Machine learning techniques for protein function prediction. Proteins: Structure, Function, and Bioinformatics 88, 397-413 (2020).

51. Jing, F., Zhao, L., Yandeau-Nelson, M.D. \& Nikolau, B.J. Two distinct domains contribute to the substrate acyl chain length selectivity of plant acyl-ACP thioesterase. Nature communications 9,860 (2018).

52. Rzhetsky, A. \& Nei, M. Theoretical foundation of the minimum-evolution method of phylogenetic inference. Mol. Biol. Evol. 10, 1073-1095 (1993).

\section{Figures}
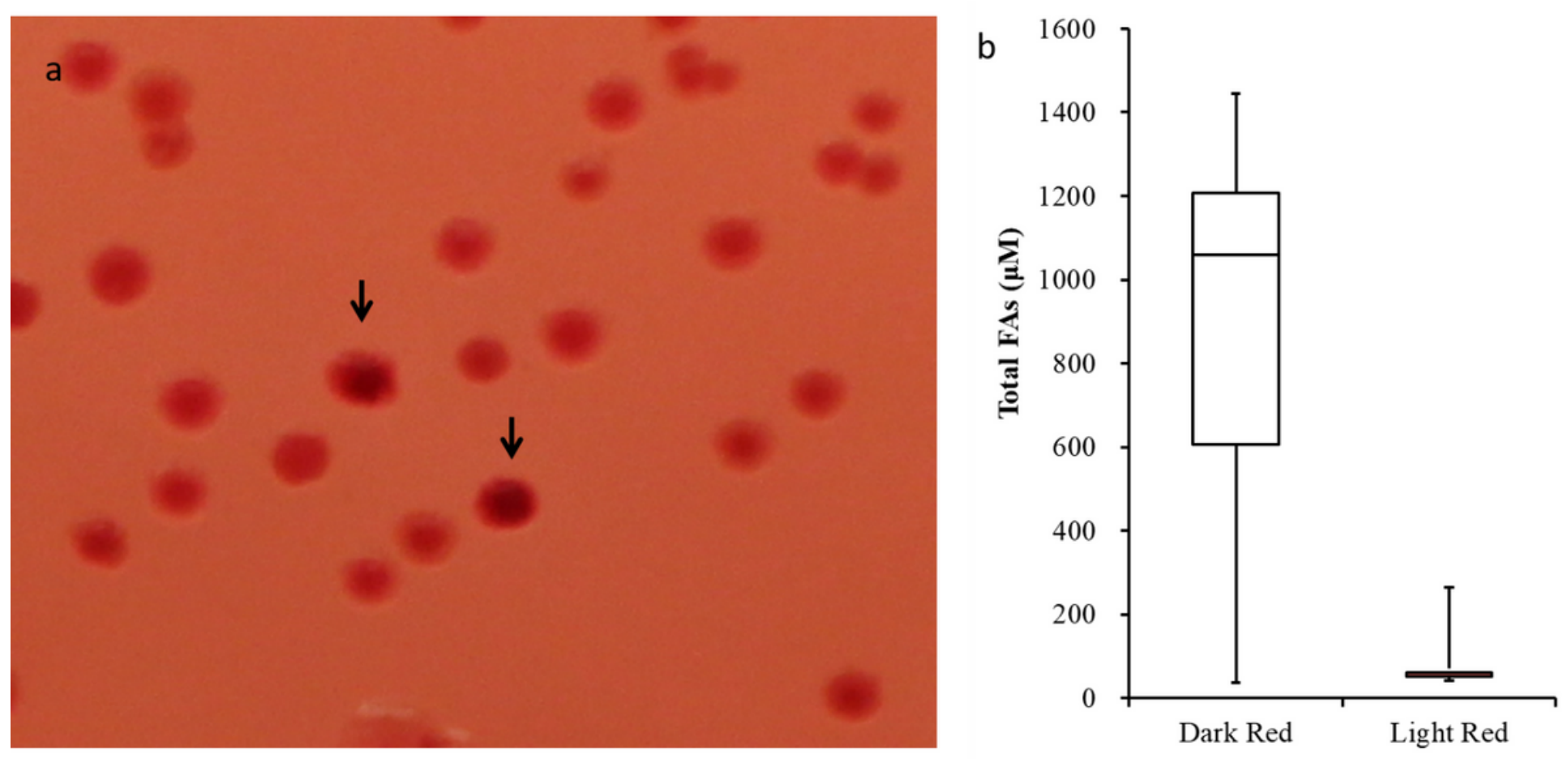

\section{Figure 1}

Efficacy of the Neutral Red plate screening assay. (a) Colonies expressing acyl-ACP TE variants were grown at $30^{\circ} \mathrm{C}$ for three days on Petri plates with media supplemented with Neutral Red dye. The colonies displaying a more intense red color are indicated by arrows. (b) Box-and-whisker plot of fatty acid productivity of cultures that were inoculated from "dark-red" $(n=177)$ and "light-red" $(n=77)$ colonies. Ttest $p$-value $<0.01$. 


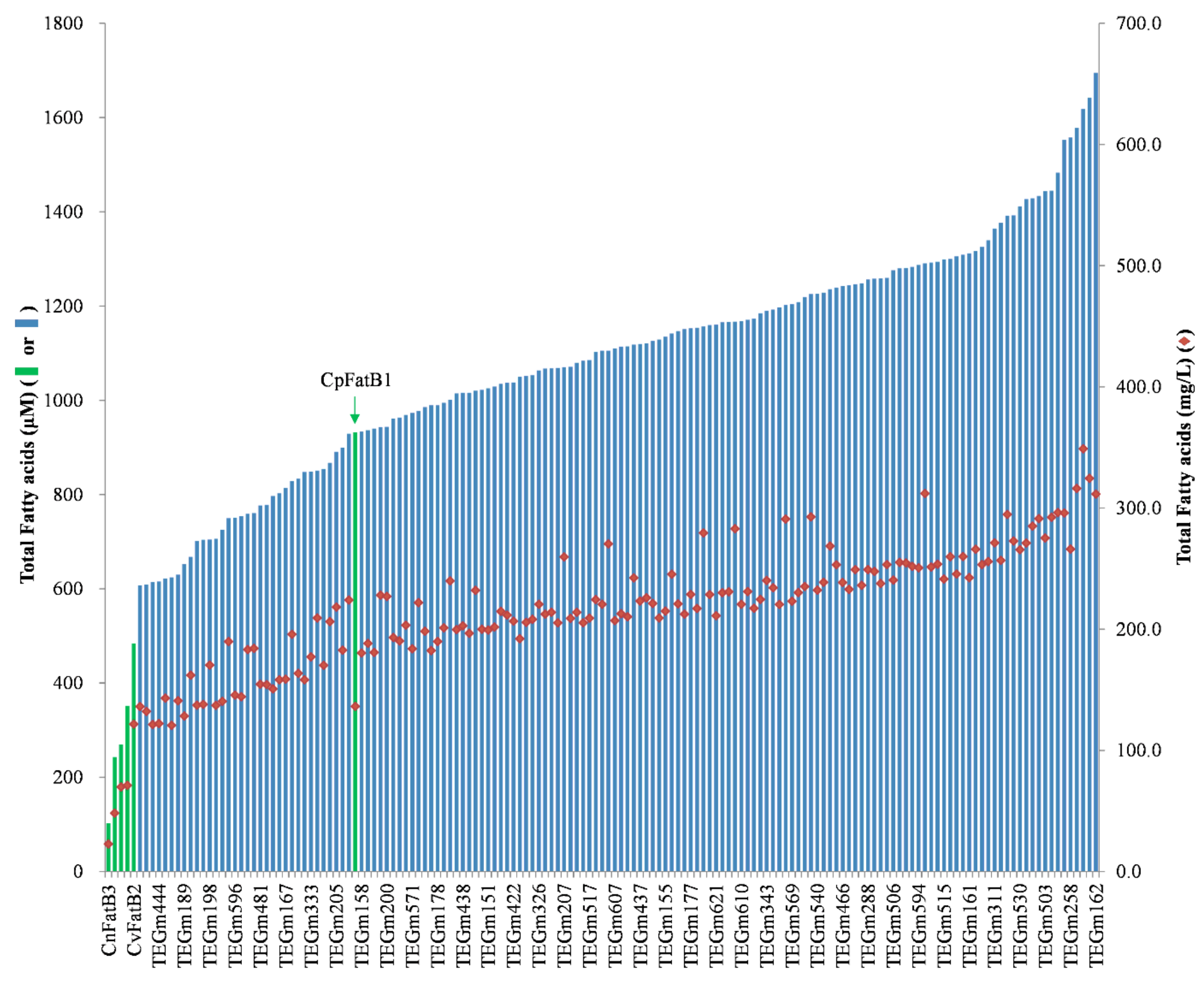

Figure 2

Fatty acid productivity of six parental acyl-ACP TEs (green data-bars) and representative acyl-ACP TE variants (blue data-bars and red diamond data-points). Data-bars represent fatty acid productivity $(\mu \mathrm{M})$, and red-diamond data-points represent fatty acids productivity $(\mathrm{mg} / \mathrm{L})$. 


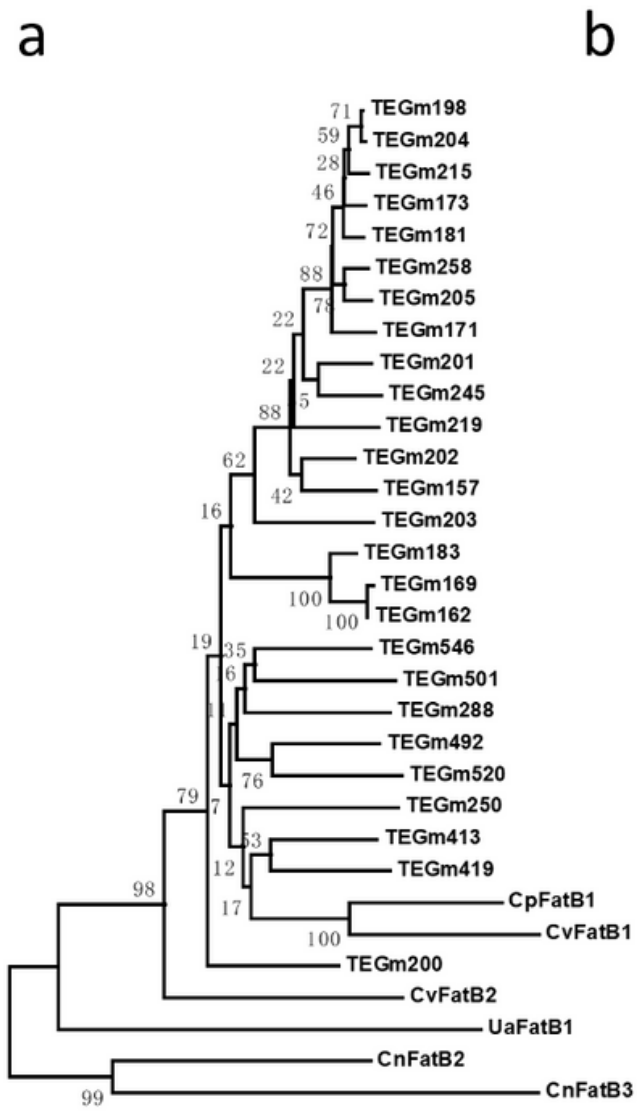

\begin{tabular}{|c|c|c|c|c|c|c|c|c|c|c|c|c|c|c|c|c|}
\hline \multirow{2}{*}{$\begin{array}{l}\text { mutant } \\
\text { names }\end{array}$} & \multirow{2}{*}{ Class $^{b}$} & \multirow{2}{*}{$\begin{array}{c}\text { Frequency in } \\
\text { sequenced samples } \\
(\text { Total }=175)\end{array}$} & \multirow{2}{*}{$\begin{array}{c}\text { Total } \\
\text { FAs(uM) }\end{array}$} & \multirow{2}{*}{$\begin{array}{c}\text { Total FAs } \\
(\mathrm{mg} / \mathrm{L})\end{array}$} & \multicolumn{12}{|c|}{ mol percentage $(\%)$} \\
\hline & & & & & $4: 0$ & 6.0 & $8: 0$ & $10: 0$ & $10: 1$ & $12: 0$ & $12: 1$ & 14.0 & $14: 1$ & $16: 0$ & $16: 1$ & 18:1 \\
\hline TEGml98 & I & 2 & 705 & 170 & 0.1 & 0.3 & 1.3 & 0.1 & 0.0 & 1.4 & 0.1 & 36.4 & 0.7 & 23.0 & 36.6 & 0.0 \\
\hline TEGm204 $4^{a}$ & I & 3 & 1203 & 291 & 0.1 & 0.2 & 1.3 & 0.1 & 0.0 & 1.6 & 0.3 & 38.0 & 1.5 & 19.8 & 33.6 & 3.4 \\
\hline TEGm215 & II & 1 & 977 & 222 & 1.6 & 1.0 & 9.0 & 0.7 & 0.1 & 5.6 & 1.7 & 30.6 & 6.5 & 15.2 & 24.3 & 3.7 \\
\hline TEGml73 & 1 & 1 & 1226 & 293 & 0.3 & 0.3 & 2.8 & 0.4 & 0.0 & 3.5 & 0.5 & 35.9 & 3.1 & 12.0 & 36.2 & 4.9 \\
\hline TEGml 81 & I & 1 & 829 & 196 & 0.4 & 0.5 & 3.7 & 0.7 & 0.0 & 4.8 & 0.5 & 35.5 & 4.6 & 15.1 & 29.2 & 5.0 \\
\hline TEGm258 & III & 1 & 1558 & 266 & 4.4 & 6.4 & 50.4 & 6.4 & 1.1 & 4.7 & 1.8 & 9.1 & 1.3 & 10.2 & 1.9 & 2.3 \\
\hline TEGm205 & I & 1 & 891 & 218 & 0.0 & 0.1 & 0.3 & 0.0 & 0.0 & 0.7 & 0.0 & 35.9 & 1.1 & 23.9 & 34.1 & 3.9 \\
\hline TEGml71 & II & 1 & 1118 & 242 & 1.3 & 1.4 & 17.6 & 0.8 & 0.1 & 6.4 & 3.1 & 26.2 & 5.9 & 12.1 & 23.0 & 2.0 \\
\hline TEGm201 & I & 1 & 594 & 148 & 0.1 & 0.2 & 1.3 & 0.1 & 0.0 & 1.2 & 0.1 & 19.3 & 1.3 & 28.4 & 41.1 & 7.0 \\
\hline TEGm245 & I & 1 & 1106 & 270 & 0.1 & 0.0 & 1.0 & 0.0 & 0.0 & 0.7 & 0.0 & 32.1 & 0.0 & 27.7 & 38.5 & 0.0 \\
\hline TEGm219 & I & 1 & 528 & 123 & 0.1 & 0.5 & 2.4 & 0.3 & 0.0 & 4.4 & 0.2 & 54.7 & 3.8 & 16.1 & 17.5 & 0.0 \\
\hline TEGm202 & I & 1 & 1001 & 240 & 0.4 & 0.4 & 2.8 & 0.3 & 0.0 & 4.0 & 0.8 & 30.1 & 2.9 & 17.9 & 36.4 & 4.0 \\
\hline TEGml57 & II & 1 & 598 & 137 & 0.4 & 1.0 & 12.0 & 0.7 & 0.0 & 4.0 & 0.5 & 30.9 & 4.6 & 19.1 & 19.2 & 7.6 \\
\hline TEGm203 & I & 1 & 526 & 127 & 0.1 & 0.1 & 0.6 & 0.1 & 0.0 & 2.4 & 0.0 & 45.1 & 2.2 & 24.1 & 19.6 & 5.7 \\
\hline TEGml 83 & II & 1 & 1236 & 269 & 0.4 & 0.5 & 12.7 & 4.2 & 0.8 & 9.2 & 6.6 & 23.2 & 11.7 & 10.0 & 18.4 & 2.4 \\
\hline TEGml69 & III & 1 & 1427 & 271 & 0.4 & 4.2 & 33.7 & 4.6 & 0.6 & 11.7 & 2.8 & 18.8 & 14.6 & 3.8 & 1.9 & 2.7 \\
\hline TEGml62 ${ }^{\mathrm{a}}$ & III & 147 & 1695 & 311 & 0.5 & 4.9 & 38.5 & 5.3 & 0.5 & 11.7 & 2.2 & 19.4 & 11.6 & 3.1 & 0.6 & 1.5 \\
\hline TEGm546 & II & 1 & 607 & 136 & 0.0 & 0.3 & 1.3 & 5.2 & 0.6 & 21.1 & 7.1 & 22.1 & 11.7 & 14.9 & 12.2 & 3.4 \\
\hline TEGm501 & I & 1 & 868 & 206 & 0.0 & 0.1 & 0.4 & 0.4 & 0.0 & 9.1 & 1.0 & 38.7 & 4.9 & 17.1 & 23.7 & 4.6 \\
\hline TEGm288 & III & 1 & 1256 & 249 & 0.2 & 0.3 & 30.9 & 5.5 & 2.2 & 9.8 & 8.5 & 12.9 & 7.1 & 12.1 & 8.3 & 2.2 \\
\hline TEGm492 & I & 1 & 604 & 146 & 0.2 & 0.2 & 2.5 & 1.5 & 0.0 & 8.6 & 0.0 & 21.9 & 2.8 & 25.7 & 24.0 & 12.5 \\
\hline TEGIm520 & II & 1 & 1021 & 232 & 0.0 & 0.2 & 2.4 & 3.1 & 1.5 & 8.8 & 10.3 & 24.0 & 15.0 & 11.4 & 20.6 & 2.8 \\
\hline TEGm250 & II & 1 & 1618 & 349 & 1.6 & 1.7 & 20.0 & 4.3 & 0.7 & 3.8 & 2.0 & 19.8 & 3.5 & 19.1 & 17.1 & 6.2 \\
\hline TEGm413 & II & 1 & 1142 & 245 & 0.1 & 0.3 & 14.8 & 6.6 & 1.4 & 13.6 & 7.6 & 14.2 & 5.9 & 14.7 & 16.9 & 3.9 \\
\hline TEGm419 & II & 1 & 1392 & 295 & 0.1 & 0.2 & 13.2 & 7.6 & 2.0 & 13.7 & 12.2 & 15.2 & 8.0 & 8.9 & 15.2 & 3.6 \\
\hline CpFatB1 & III & & 932 & 136 & 0.01 & 1.51 & 93.46 & 2.18 & 0.44 & 0.87 & 0.21 & 0.00 & 0.10 & 1.21 & 0.00 & 0.00 \\
\hline CvFatB1 & III & & 243 & 48 & 0.00 & 1.22 & 24.16 & 21.59 & 1.48 & 4.84 & 3.89 & 21.10 & 0.21 & 10.17 & 5.60 & 5.74 \\
\hline TEGm 200 & I & 1 & 944 & 227 & 0.3 & 0.0 & 1.8 & 0.4 & 0.1 & 4.7 & 3.7 & 19.2 & 8.3 & 16.6 & 41.5 & 3.5 \\
\hline CvFatB2 & I & & 484 & 122 & 0.00 & 0.00 & 0.67 & 0.56 & 0.15 & 0.31 & 0.24 & 27.85 & 0.00 & 39.95 & 11.22 & 19.06 \\
\hline UaFatB1 & III & & 352 & 71 & 0.07 & 0.31 & 21.79 & 19.72 & 0.93 & 3.56 & 1.95 & 32.23 & 0.27 & 8.37 & 6.27 & 4.54 \\
\hline CnFatB2 & I & & 270 & 70 & 0.00 & 0.00 & 0.14 & 0.00 & 0.00 & 0.00 & 0.00 & 13.77 & 0.00 & 52.57 & 7.45 & 26.08 \\
\hline CnFatB3 & II & & 103 & 23 & 0.00 & 0.12 & 6.02 & 0.00 & 0.00 & 34.29 & 2.28 & 26.17 & 5.71 & 12.17 & 3.96 & 9.27 \\
\hline
\end{tabular}

$\stackrel{\mapsto}{0.02}$

Figure 3

Fatty acid productivity and fatty acid specificity of evolved acyl-ACP TE variants. (a) Dendrogram representation of sequence similarities among acyl-ACP TE variants. The dendrogram was inferred using the Minimum Evolution method 52. The bootstrap consensus tree (bootstrap value identified at each node) was inferred from 250 replicates and it represents the evolutionary history of each acyl-ACP TE. (b) Fatty acid profiles of 26 unique acyl-ACP TE variants generated in this study and compared to the six parental acyl-ACP TEs used to constrain the directed evolution strategy. The intensity of the green shading of each cell is proportional to the mol \% of each fatty acid. a Among the 175 acyl-ACP TE variants recovered in this study, the TEGm2198, TEGm204 and TEGm162 variants recurred 2, 3 and 147 times, respectively. b Acyl-ACP TEs can be classified into three groups based on their substrate specificity: Class I enzymes primarily hydrolyze acyl-ACPs of 14- and 16-carbon fatty acyl chains, Class II enzymes prefer 8- to 16-carbon acyl-chains, and Class III enzymes have a preference for 8-carbon acyl-chains. 

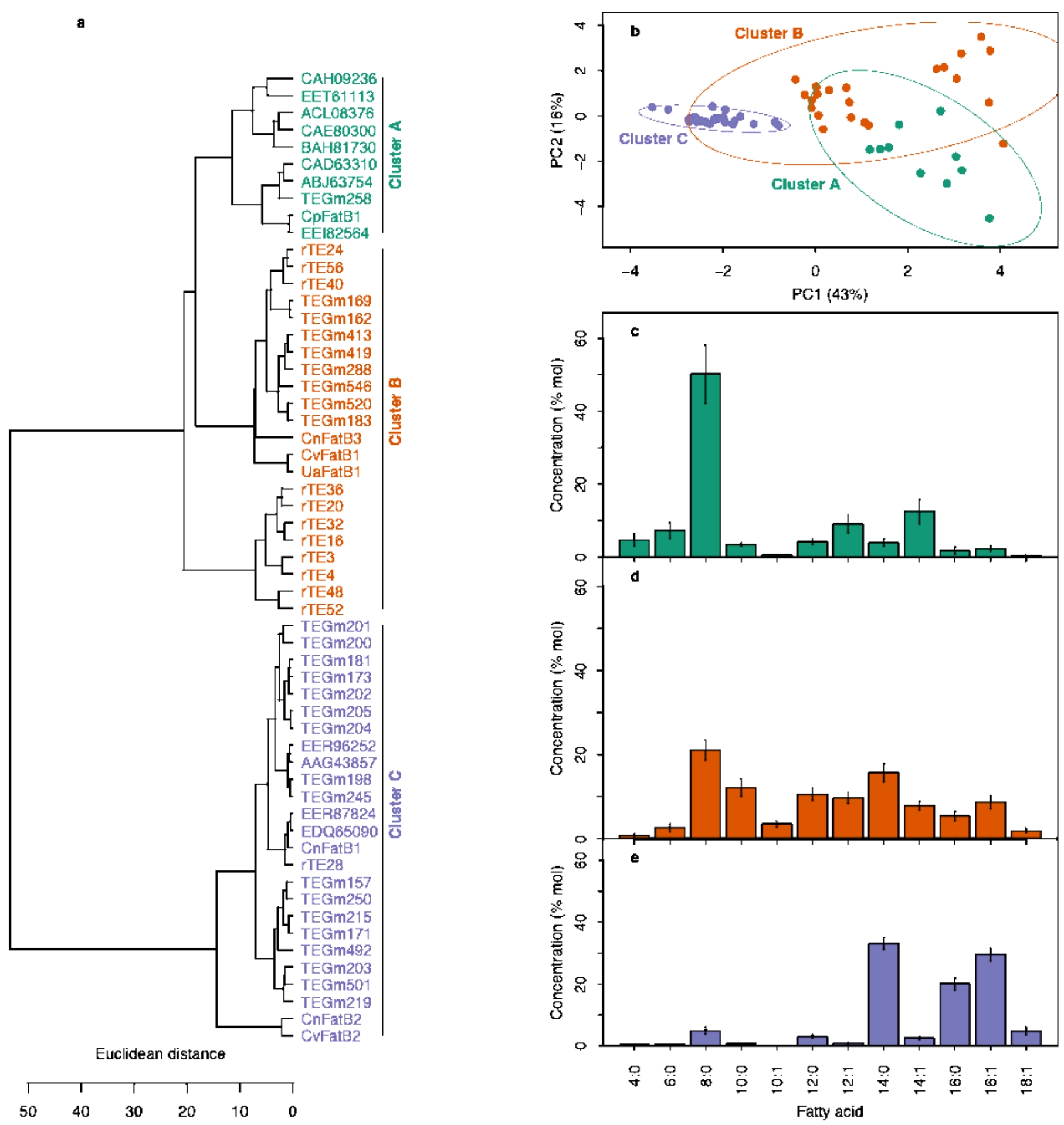

Figure 4

Categorizing acyl-ACP TEs based on fatty acid productivity profiles. (a) Enzyme cluster membership was determined by hierarchical clustering of fatty acid profiles produced when each acyl-ACP TE was expressed in E. coli. (b) The PCA model based on the fatty acid profiles produced when each acyl-ACP TE was expressed in E. coli. The model explains $59 \%$ of the data variation, and segregates the 57 enzymes into three clusters demonstrated by $95 \%$ confidence ellipses. (c) The fatty acids profiles produced by acyl- 
ACP TEs that belong to Cluster A. (d) The fatty acids profiles produced by acyl-ACP TEs that belong to Cluster B. (e) The fatty acids profiles produced by acyl-ACP TEs that belong to Cluster C.
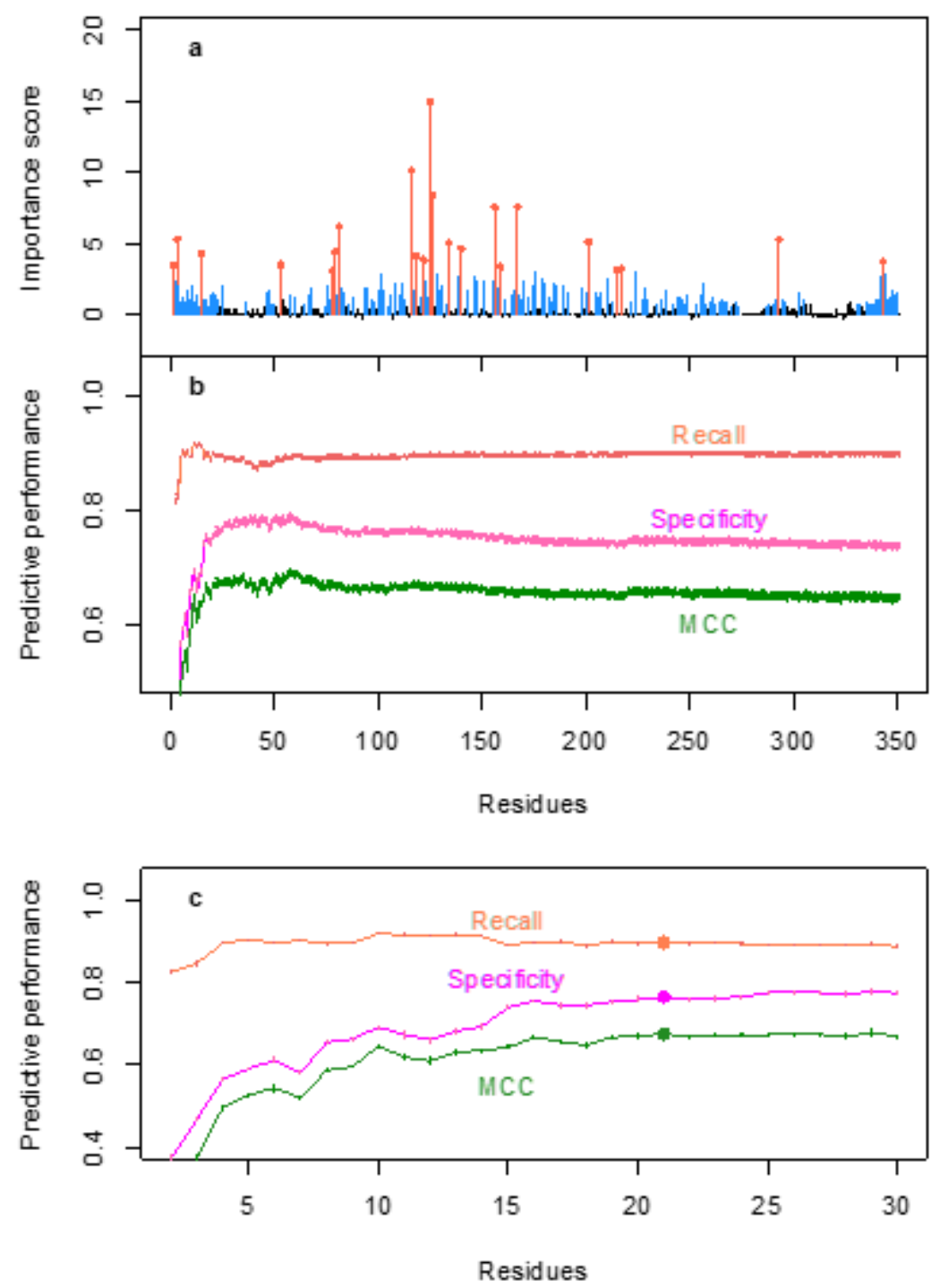

\section{Figure 5}

Identification of residue positions predicted to govern acyl-ACP TE substrate specificity. (a) The importance scores for each residue position were generated by the random forest model that uses all 350 positions and one random variable as the predictors. The most impactful positions that determine the substrate specificity of the enzyme (orange-colored data points) were identified via IFS and have a qvalues $<0.001$. Non-significant positions are in black. (b) IFS selects the most important predictor set by evaluating the predictive performance of the associated model. (c) A zoom-in view of the predictive performance evaluated by IFS. MCC hits the plateau when top 22 residue positions (highlighted by filled circles) are included in the model. 


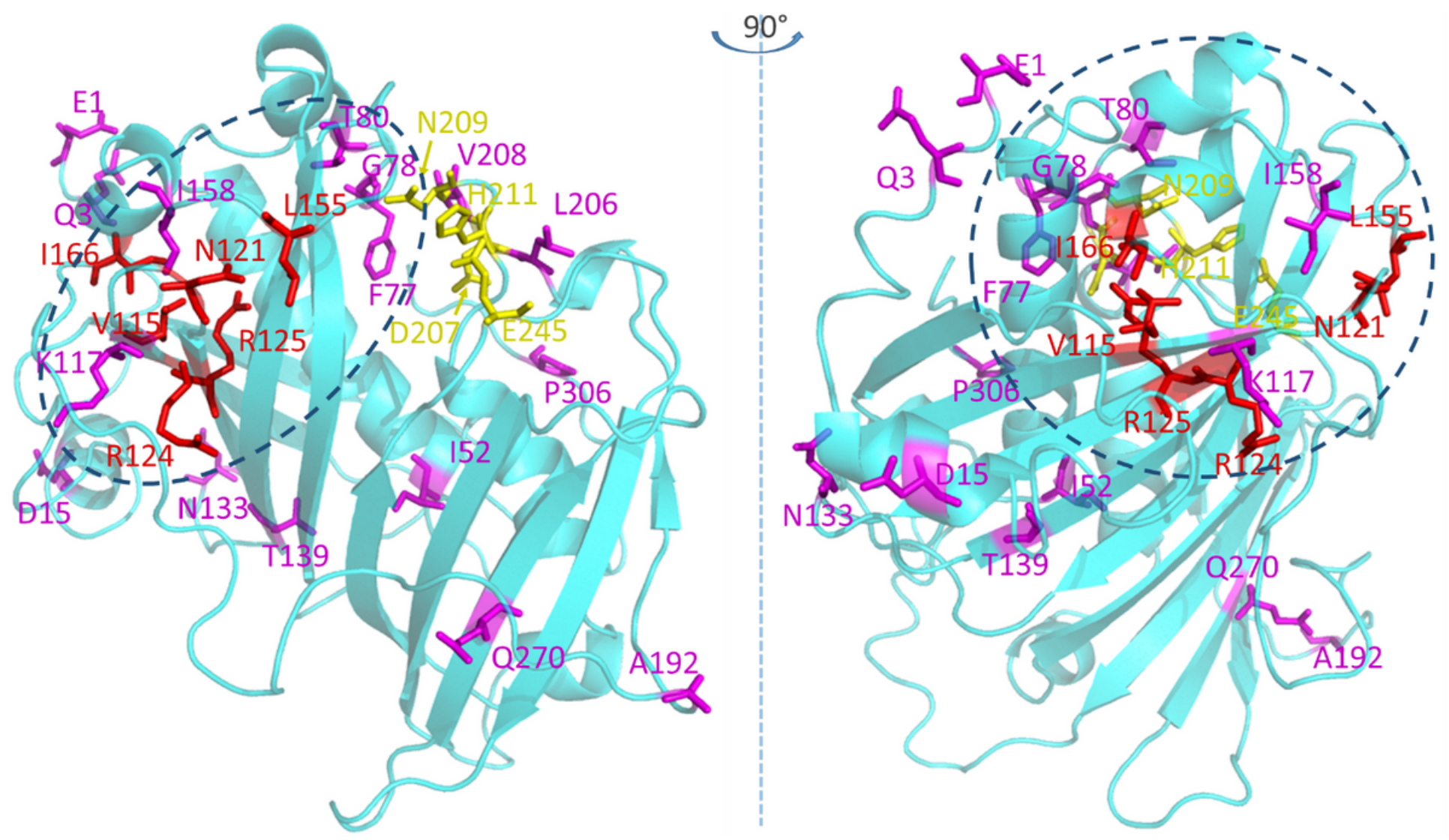

Figure 6

Residues that are significant in determining the substrate specificity of acyl-ACP TE. Twenty two residues selected by random forest classifier (Figure 6) are shown as stick models. Red colored residues have previously been experimentally verified to affect substrate specificity51. Catalytic residues are shown in yellow. The dotted ovals indicate the structural region where the substrate binding pocket is located1.

\section{Supplementary Files}

This is a list of supplementary files associated with this preprint. Click to download.

- SupplementalTable1.pdf

- SupplementalTable2.pdf

- SupplementalTable3.pdf

- SupplementalTable4a.pdf

- SupplementalTable4b.pdf

- SupplementalFigures.docx 